\title{
Modeling of nonstationary electron precipitation by the whistler cyclotron instability
}

\author{
A. G. Demekhov ${ }^{1}$, A. A. Lyubchich ${ }^{2}$, V. Y. Trakhtengerts ${ }^{1}$, E. E. Titova ${ }^{2}$, J. Manninen ${ }^{3}$, T. Turunen ${ }^{3}$ \\ ${ }^{1}$ Institute of Applied Physics, Nizhny Novgorod, Russia \\ ${ }^{2}$ Polar Geophysical Institute, Apatity, Russia \\ ${ }^{3}$ Sodankylä Geophysical Observatory, Sodankylä, Finland
}

Received: 3 September 1997 / Revised: 3 February 1998 / Accepted: 10 March 1998

\begin{abstract}
We study a simple self-consistent model of a whistler cyclotron maser derived from the full set of quasi-linear equations. We employ numerical calculations to demonstrate dependencies of pulsation regimes of whistler-mode wave interactions with energetic electrons on plasma parameters. Possible temporal evolution of those regimes in real conditions is discussed; calculations are compared with case-study experimental data on energetic electron precipitation pulsations. A reasonable agreement of the model results and the observations has been found.
\end{abstract}

Key words. Magnetospheric physics (Auroral phenomena; Energetic particles, precipitating; Storms and substorms)

\section{Introduction}

It is well known that the whistler cyclotron instability (WCI) is an important agent of energetic electron precipitation from the radiation belts. One of its interesting features is existence of nonstationary (pulsating) regimes in presence of a constant free energy source which may be, e.g., due to drift of injected energetic electrons into an instability region or local acceleration mechanisms. These pulsations can be damped or undamped and they are believed to account for some types of experimentally observed phenomena such as pulsating auroral patches (for a review of observations see, e.g., Sandahl (1984), theoretical investigation has been reported by Trakhtengerts et al. (1986), Davidson and Chiu (1986), Demekhov and Trakhtengerts (1994)).

Due to the complexity of the self-consistent theory of WCI it seems fruitful to employ simplified models which enable a quick means of obtaining estimations and thus rapid choice of parameters that provide the best agreement with the experiment. Such models have been suggested and analyzed, e.g., by Bespalov and Trakhtengerts (1976), Schulz (1974), Davidson (1979), Bespalov (1981), and Davidson and Chiu (1986). In particular, undamped pulsating solutions in presence of a constant free energy source have been obtained by Bespalov (1981), and Davidson and Chiu (1986). Bespalov (1981) used a rigorous approach based on expanding the pitch-angle distribution function over a set of eigenfunctions of the quasi-linear diffusion operator and obtained a set of ordinary differential equations (ODEs) valid for weak pitch angle diffusion regime. Davidson and Chiu (1986) adopted a phenomenological approach aiming to investigate the moderate and strong diffusion regimes. However, as shown by Trakhtengerts et al. (1986), Demekhov (1991), Demekhov and Trakhtengerts (1994), the distribution function of energetic electrons can strongly vary in these regimes, as well as wave spectrum shape, so using a full pitch-angle distribution seems necessary for proper treatment of the loss cone filling effect in the case of moderate diffusion.

The study reported in this work is motivated by observations of auroral electron precipitation pulsations with periods about $T \sim 10^{2} \mathrm{~s}$, for which satellite data on trapped energetic electrons and EISCAT data on the ionosphere ionization have been recently analyzed (Manninen et al., 1996; Lyubchich et al., 1996). For the time scales mentioned, weak pitch-angle diffusion approximation is suitable. Thus we use the rigorous model for non-stationary WCI regimes suggested by Bespalov (1981), which is based on the multi-level set of equations for the WCI taking into account nonlinear modulation of the pitch-angle distribution of trapped energetic particles. It allows us to relate the parameters of WCI regimes to the quantities derived from observations, such as density, energy and pitch-angle distribution of energetic electrons and cold plasma density.

To make the model closer to the experiment, its generalized version with slow time dependence of the 
coefficients is considered. Such a dependence can be, e.g., due to variation of the number density, energy or anisotropy of energetic particles or background plasma density in the instability region. On the base of numerical calculations, we investigate the quantitative and qualitative dependencies of characteristics of energetic electron precipitation pulsations on those parameters and their possible temporal evolution.

An attempt is made to compare the results obtained with observational data. We try to simulate the precipitation events reported by Manninen et al. (1996), Lyubchich et al. (1996). We show that using the multilevel WCI equations, the qualitative difference of precipitation time patterns between these events can be consistently attributed to the difference in the recorded plasma parameters.

\section{Basic equations and their properties}

We start from the multi-level equations for the whistler cyclotron instability (Bespalov, 1981) taking into account nonlinear modulation of the pitch-angle distribution of trapped energetic particles. The detailed derivation of these equations can be found in the review paper by Bespalov and Trakhtengerts (1986). They have the form:

$$
\begin{aligned}
\mathrm{d} F_{i} / \mathrm{d} t & =-D_{0} \mathscr{E} \delta_{i} F_{i}+I_{i}, \\
\mathrm{~d} \mathscr{E} / \mathrm{d} t & =\left(\sum_{i} h_{i} F_{i}-v\right) \mathscr{E} .
\end{aligned}
$$

Here $\mathscr{E}$ is the whistler wave energy density; $F_{i}$ and $I_{i}$ $(i=1,2, \ldots)$ are the amplitudes in the expansion of the pitch-angle distribution function of energetic electrons $F(\theta)$ and the source function $I(\theta)$ into a complete set of eigenfunctions $Z_{i}$ of the quasi-linear diffusion operator:

$$
\begin{gathered}
F(\theta)=\sum_{i} F_{i} Z_{i}(\theta), \\
I(\theta)=\sum_{i} I_{i} Z_{i}(\theta), \\
\frac{\mathrm{d}}{\mathrm{d} \eta} \eta \frac{\mathrm{d} Z_{i}}{\mathrm{~d} \eta}=-\delta_{i} Z_{i},
\end{gathered}
$$

$\eta=\sin ^{2} \theta, \theta$ is the equatorial pitch angle, $\delta_{i}$ are the corresponding eigenvalues; $h_{i}$ are the coefficients of the growth rate expansion; $v$ is the wave energy damping rate; and $D_{0} \approx \omega_{B L} \beta_{*} / N_{c} W$ where $\beta_{*}=\omega_{p L}^{2} v^{2} / \omega_{B L}^{2} c^{2}$, $\omega_{p L}$ and $\omega_{B L}$ are the electron plasma frequency, and gyrofrequency, respectively, index $L$ refers to the equatorial plane, $W=m v^{2} / 2$ is the electron energy, $N_{c}$ is the cold plasma number density. This system of equations can be derived from the general quasi-linear theory if (1) diffusion over energy is neglected; (2) wave energy spectrum is narrow in frequency; and (3) weak pitch-angle diffusion regime is realized. The 1st and the 2nd assumptions are valid in regions with sufficiently high background plasma density, when the frequency of whistler waves is low, $\omega \sim \omega_{B L} / \beta_{*} \ll \omega_{B L}$. Weak pitchangle diffusion regime exists for sufficiently low values of the wave energy density. Quantitative criterion for this can be written as
$D_{0} \mathscr{E} \delta_{1} \ll 2 / T_{b}$,

where $T_{b}$ is the particle bounce period; this inequality means that emptying of the loss cone is much faster than its filling by the pitch angle diffusion.

The boundary conditions for the eigenvalue problem (3) correspond to the weak pitch-angle diffusion regime:

$Z_{i}\left(\eta_{c} \equiv æ_{c}^{2}\right)=0, \quad Z_{i}^{\prime}\left(\eta_{m} \equiv æ_{m}^{2}\right)=0$,

$æ_{c}=\sin \theta_{c}=\sigma^{-1 / 2}$ is the sine of equatorial loss cone angle ( $\sigma$ is the mirror ratio); $æ_{m}$ is the upper boundary of the interaction region, $x_{m} \approx \sqrt{1-\left(4 \beta_{*}\right)^{-1}}$. For particles with $æ>x_{m}$, the condition of cyclotron resonance with parallel propagating whistler waves with $\omega<\omega_{B L} / 2$ cannot be satisfied.

The eigenfunctions and eigenvalues of Eq. (3) can be expressed in terms of Bessel functions. This is readily seen by applying the substitution $x=2 \delta_{i}^{1 / 2} æ=2\left(\delta_{i} \eta\right)^{1 / 2}$. Writing down the solution for the resulting Bessel equation and using the boundary conditions, one can see (Bespalov, 1981) that the eigenvalues $\delta_{i}$ are the roots of the following characteristic equation:

$J_{0}\left(x_{c}^{(i)}\right) Y_{1}\left(p x_{c}^{(i)}\right)-Y_{0}\left(x_{c}^{(i)}\right) J_{1}\left(p x_{c}^{(i)}\right)=0$,

where $x_{c}^{(i)}=2 \delta_{i}^{1 / 2} æ_{c}, p=æ_{m} / æ_{c}$. The eigenfunctions $Z_{i}$ have the form

$Z_{i}=C_{i}\left[J_{0}\left(x_{c}^{(i)}\right) Y_{0}(x)-Y_{0}\left(x_{c}^{(i)}\right) J_{0}(x)\right]$,

$C_{i}=\pi \delta_{i}, x=2 \delta_{i}^{1 / 2} æ$. The coefficients $h_{i}$ are written as

$h_{i}=\left(\omega_{B L} / N_{c}\right)\left[\Re_{m}^{2} Z_{i}\left(x_{m}^{(i)}\right)-1\right]$.

For practical usage, one can solve numerically the characteristic Eq. (6) for a range of values of $p$, to tabulate dimensionless roots $x_{c}^{(i)}$ as dependent of $p$; these results can then be used for different combinations of $æ_{c}$ and $æ_{m}$.

Analysis of the system (1) with constant coefficients showed (Bespalov, 1981) that it can have oscillatory solutions corresponding to pulsating precipitation of energetic electrons. To produce pulsating regimes, it is necessary to consider at least two components in the distribution function expansion $(i=1,2)$; if we take only one component, we obtain only damped pulsations (Bespalov and Trakhtengerts, 1976; Davidson, 1979). Further we take into account only the first two components: this is possible because the eigenvalues $\delta_{i}$ rapidly increase with the eigennumber $i$. In particular, $\delta_{2} / \delta_{3} \simeq 0.3$ for $æ_{c} \approx 0.1$ which corresponds to $L \approx 5$. In the quasistationary case, $F_{i} \approx I_{i} /\left(D_{0} \mathscr{E} \delta_{i}\right)$. Thus if $I_{3} \sim I_{2}$, then $F_{3} / F_{2} \sim I_{3} \delta_{2} / I_{2} \delta_{3} \sim 0.3$, and the contributions of $F_{3}$ and $F_{2}$ to the growth rate will compare as for $\left|h_{3} F_{3} / h_{2} F_{2}\right| \sim 0.3\left(h_{2} \simeq h_{3}\right)$. On the other hand, similar comparison of the roles of the 1st and 2nd components shows that if $I_{1} \sim I_{2}$, then $\left|h_{2} F_{2} / h_{1} F_{1}\right| \sim 3.5\left(\delta_{1} / \delta_{2} \sim 25\right.$, $\left.\left|h_{2} / h_{1}\right| \sim 85\right)$. This means that the relative role of the term $F_{2}$ as compared with $F_{1}$ is 10 times as significant as the role of $F_{3}$ as compared with $F_{2}$. Omission of terms $\left\{F_{i}: i>2\right\}$ can be justified also qualitatively: solution of the full quasi-linear equations does not show additional strong peculiarities of the temporal regime of the 
instability except the pulsating regime (e.g., Demekhov and Trakhtengerts, 1994) which can be obtained using the terms $F_{1}$ and $F_{2}$ in the expansion of $F(\theta)$.

We note that the basic eigenfunction $Z_{1}(\eta)$ monotonically increases with $\eta$, and higher eigenfunctions $Z_{i}$ oscillate near zero crossing the $\eta$ axis $(i-1)$ times within the interval $\left.\eta \in] \eta_{c} ; \eta_{m}\right]$. When the amplitude $F_{2}$ of the 2nd "harmonic" $Z_{2}$ increases, the pitch-angle distribution changes its shape from a monotonically growing function to a nonmonotonous one (Fig. 1), that can be described as butterfly-like because it reaches maximum at a given pitch angle.

\subsection{Parameters of pulsations}

It is interesting to discuss characteristics of the pulsating regimes under realistic assumptions of cold and hot plasma parameters. These parameters are usually not constant in time, thus we should consider possible changes in the cyclotron instability regime by suitable variation of the coefficients in Eq. (1). In this study we will assume sufficiently slow evolution of the external parameters, so that we can use the same equations with correspondingly varying coefficients.

Qualitatively, consequences of variation of each parameter can be understood based on known analytical and numerical results (Bespalov and Trakhtengerts, 1986; Demekhov and Trakhtengerts, 1994):

1. The duration of an impulse $\tau_{i}$ is proportional to the wave growth time which is of order of $v^{-1}$.

2. The period of pulsations is the sum of $\tau_{i}$ and the pause duration $\tau_{p}, T=\tau_{i}+\tau_{p} \sim \tau_{p}$, where $\tau_{p}$ is determined by the time $\tau_{I}$ of energetic particle accumulation to the instability threshold. Thus $\tau_{p}$ depends mainly on the particle source intensity and anisotropy and on wave damping $v$ $\left(\tau_{p} \sim \tau_{I} \propto\left(\sum_{i} h_{i} I_{i} / v\right)^{-1}\right)$; also it weakly depends on $N_{c}$ and $W$. If the energetic particle source is due to their drift through the interaction region, the source intensity is proportional to $N_{h} / t_{D}$ where $N_{h}$ is the

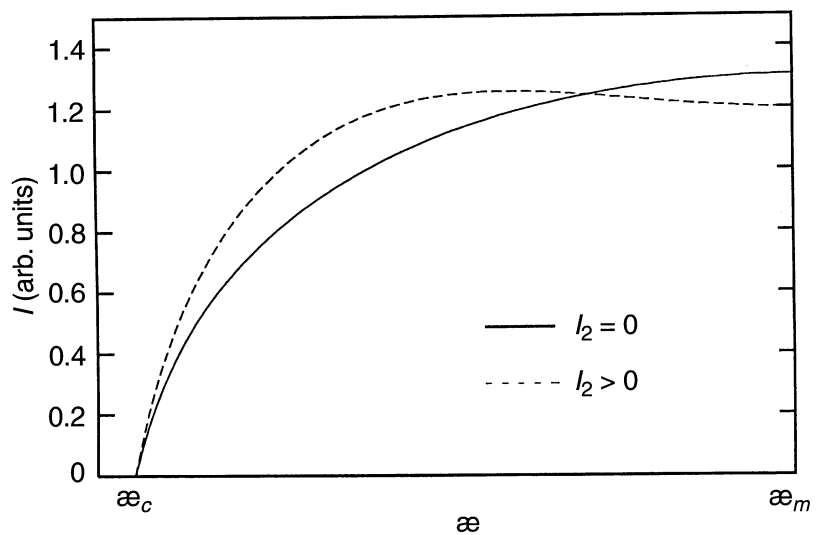

Fig. 1. Pitch-angle dependence of the energetic electron source: two harmonics, $Z_{1,2}$, are taken into account. When $I_{2}=0$, the distribution is monotonously growing; otherwise it can have a maximum at some point $æ$ between $æ_{c}$ and $æ_{m}$ number density in a cloud at the boundary of the interaction region, and $t_{D}$ is the drift time.

Conditions for a pulsating regime in the system (1) are as follows:

1. The dimensionless parameter $\alpha=\left(v \tau_{I}\right)^{-1}$ has to be sufficiently small, and

2. The 2-nd harmonic $I_{2}$ in the source function expansion (2) has to be large enough (Bespalov, 1981).

The damping rate $v$ is determined by the effective reflection coefficient $R$ according to the formula $v=2|\ln R| / T_{g}, T_{g}=\oint \mathrm{d} z / v_{g}$ is the period of wave packet oscillations between conjugate ionospheres. $R$ depends on ionospheric conductivity and on parameters of magnetospheric cold plasma since damping of whistler waves is due to partial reflection from the ionosphere and from the duct ends and also due to refraction losses. The value of $R$ is a rather important parameter for our problem, since it influences the period of pulsations; however its exact value is unknown, so we can use only rough estimations based on ray-tracing studies.

We will consider the temporal variations that can occur during a passage of a cloud of energetic electrons through the cyclotron interaction region. In this case we can expect variation of the energetic particle source amplitude (which is proportional to the electron density in a cloud), pitch-angle anisotropy, and characteristic energy of electrons. Other quantities that can vary are: background plasma density $N_{c}$ and effective reflection coefficient $R$.

The most substantial variation can be expected in energetic electron density and anisotropy. The "sloshing" or "butterfly" component of the pitch angle distribution of the injected particles, which is due to the 2-nd harmonic $I_{2}$ of the source function (Fig. 1), will increase with time if the injection region is localized at a distance from the instability region. This is in agreement with recent analyses of substorm injection events (Manninen et al., 1996; Lyubchich et al., 1996). Relevant properties of pulsating regimes of CI are:

1. Increase of the source power leads to a decrease of the pulsating period $T$ and further to a transition to a damped pulsation regime.

2. The amplitude of the 2nd pitch-angle harmonic, $I_{2}$, in the energetic particle source distribution, must be above some threshold to provide existence of undamped pulsating regime of WCI. Increase of $I_{2}$ above this threshold leads to an increase of $T$.

\section{Calculation results}

For the numerical calculations, we assume that the source intensity $J \equiv I_{1}+I_{2}$ and the ratio $A=I_{2} / I_{1}$ vary according to the equations

$$
\begin{aligned}
I & =I_{0}\left[1+\Delta_{I} \sin \left(\pi t / 2 T_{I}\right)\right] \\
A & =A_{0}\left[1+\Delta_{A} \sin \left(\pi t / 2 T_{I}\right)\right]
\end{aligned}
$$

The electron energy changes linearly during each run from the value $W_{0}$ to $W_{1}$. Thus we can investigate the 


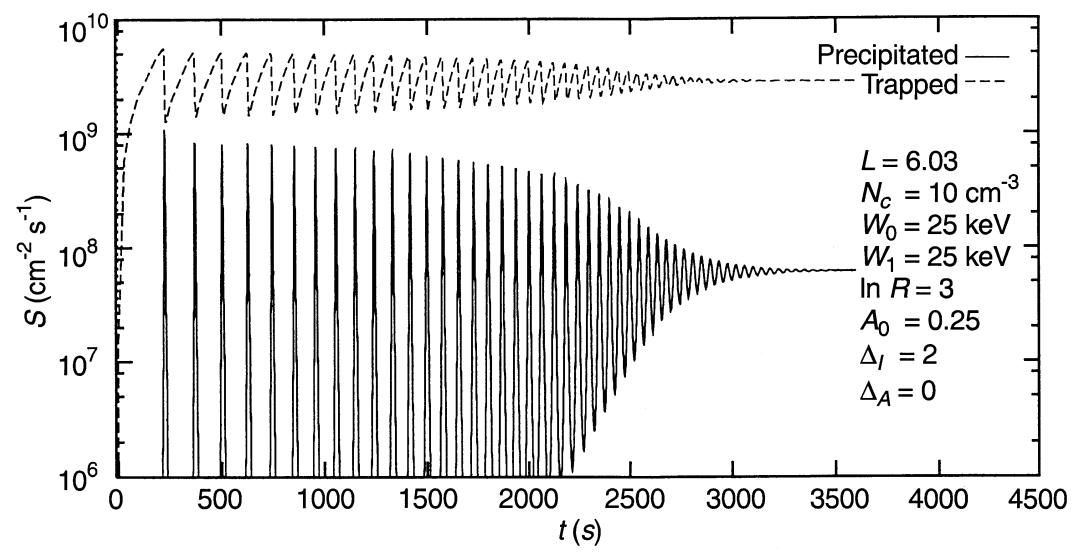

Fig. 2. Result of numerical calculations of Eq. (1) with variable intensity of the energetic particle source: Eq. (9), $T_{I}=7200 \mathrm{~s}$ influence of the most significant parameters on dynamical regimes that exist in the cyclotron maser described by Eq. (1). The restriction that the parameters $A$ and $I$ vary with the same time scale could be omitted by choosing a different temporal dependence; for the goals of this work this restriction is not significant.

Figures 2-5 show the numerical results; fluxes of trapped and precipitated energetic particle fluxes $S_{\text {tr }}$ and $S_{\mathrm{pr}}$ are plotted as functions of time. They are calculated according to the formulas

$$
\begin{aligned}
S_{\mathrm{tr}} & =v \int F(\eta)(1-\eta)^{1 / 2} \mathrm{~d} \eta \\
& =v \int \sum_{1}^{2} F_{i} Z_{i}(\eta)(1-\eta)^{1 / 2} \mathrm{~d} \eta, \\
S_{\mathrm{pr}} & =\pi \sigma v T_{b} D_{0} \mathscr{E} \sum_{1}^{2} \delta_{i} F_{i}
\end{aligned}
$$

and represent total fluxes integrated over solid angle and energy. In these equations, as earlier, $\sigma$ is the mirror ratio $v$ is the energetic electron velocity, and $T_{b}$ is the bounce period.

Figures 2 and 3 illustrate the cases when only a single parameter changes. In particular, the source intensity $I$ is variable in Fig. 2 and the pitch-angle distribution, quantified by the ratio $A$, is variable in Fig. 3; values of parameters chosen can be seen in figure legends. These figures demonstrate in a pure form, the most important features mentioned already:

1. In Fig. 2, one can see the effect of gradual increase of the source intensity from the start to the end of the run; strong pulsations exist in the beginning, when the value of $I$ is minimal; their period decreases with time while $I$ increases, then a transition to damped pulsations and further to a steady state occurs.

2. In Fig. 3, the parameter $A$, representing the "sloshing" component of the pitch-angle distribution (Fig. 1), increases during the run according to Eq. (10) (see the figure legend for parameters). Here the initial conditions have been chosen close to the stationary state, and the initial value of $A$ close to the threshold mentioned in the preceding section. According to this, small pulsations occur near $t=0$, and with increase of $A$ we see the buildup of strong pulsations whose period increases.

Figures 4 and 5 show two examples of numerical solution of the Eq. (1) which demonstrate the evolution of pulsation regimes of a cyclotron maser due to variation of both the energetic particle density and pitch angle distribution (see figure legends for parameters). Figure 4 corresponds to a slower variation $\left(T_{I 1}=3600 \mathrm{~s}\right)$ with larger change in the pitch-angle distribution $\left(\Delta_{A 1}=4\right)$ and smaller variation of the source intensity $\left(\Delta_{I 1}=2\right)$ as compared with the param-

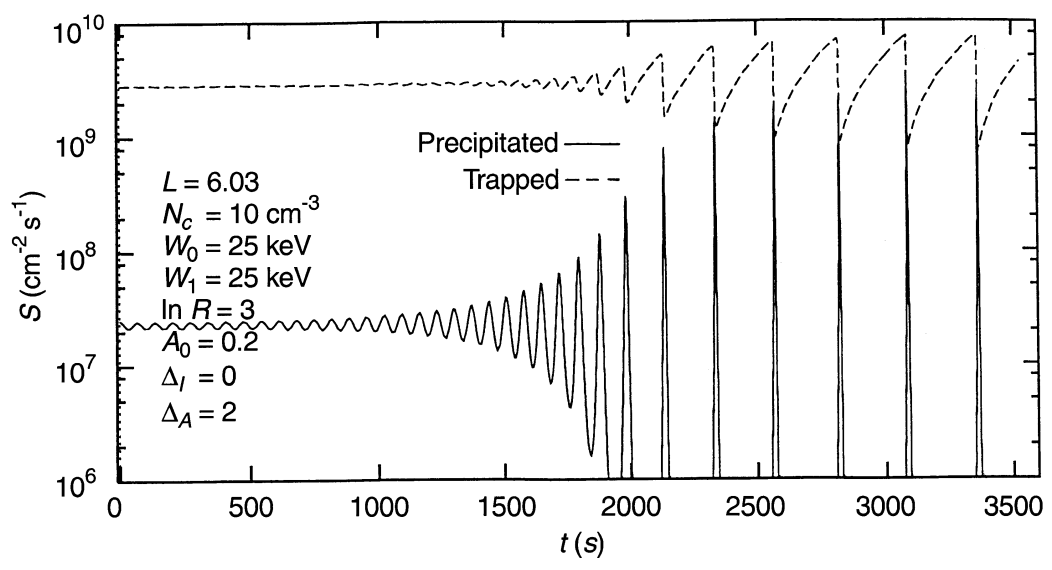

Fig. 3. Result of numerical calculations of Eq. (1) with variable pitch-angle distribution of the energetic particle source: Eq. (10), $T_{I}=7200 \mathrm{~s}$ 


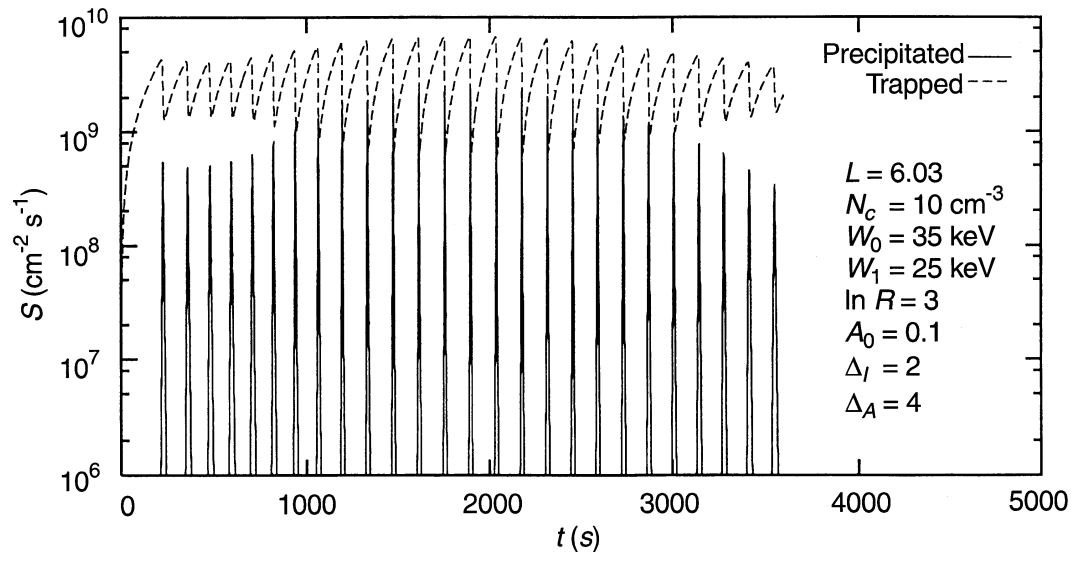

Fig. 4. Result of numerical calculations of Eq. (1) with parameters corresponding to a substantial variation of the pitch-angle distribution in the source; $T_{I}=3600 \mathrm{~s}$ eters chosen for Fig. $5\left(T_{I 2}=1800 \mathrm{~s}, \Delta_{A 2}=2\right.$, and $\Delta_{I 2}=4$ ).

From the numerical results, we can see that qualitatively different temporal evolution of pulsating regimes of CI can be seen depending on the parameters of the injected energetic electron cloud.

In particular, increase of the 2nd harmonic in the energetic electron pitch-angle distribution can result in a net increase of the pulsation period even if the simultaneous growth of the energetic electron density acts to decrease the period (Fig. 4).

Figure 5 shows that pulsating precipitation can cease when the energetic electron density is at maximum. It is very interesting to note that pulsations are not reestablished in the end of the run, though all parameters of the energetic particle source return to their initial values. This is due to the hard regime of pulsation excitation which is confirmed by the calculations not shown here. For the chosen parameters, undamped pulsations in the cyclotron maser exist only if the initial state is far enough from the stationary one, otherwise they are damped.

\section{Discussion}

The parameters of energetic electrons for numerical calculations shown in Fig. (4) and (5) were chosen so as to reproduce some features from case studies of two substorm injections accompanied by pulsating electron precipitation (Manninen et al., 1996; Lyubchich et al., 1996). Now we try to compare the calculation results with the observations. Table 1 summarizes the observation results for two events whose features are represented by calculations. We note that not all the plasma parameters necessary for the model are known. In particular, period of pulsations is determined by the drift time of energetic electrons through the interaction region (Trakhtengerts et al., 1986; Demekhov and Trakhtengerts, 1994); this time is not known since there is no information on spatial characteristics of pulsations (no optical data are available for the events discussed). Thus we had to choose the source intensity $I$ which provided the observed periods of pulsations. We can estimate the size of the interaction region using the formula $d \simeq v_{D} T$; for electron energies $W \approx 20-30 \mathrm{keV}$, observed pulsation periods $T \sim 100 \mathrm{~s}$ imply the value $d \sim 100 \mathrm{~km}$ at ionospheric level.

One can see that the main differences between the events discussed are duration of injection events, characteristics of energy and pitch-angle dispersion of the energetic electrons, and duration of precipitation pulsations. From the difference in energy and pitch-angle dispersion, we may assume that event 2 which indicates no dispersion in energy and pitch angle corresponds to an injection very close to the observation point, whereas

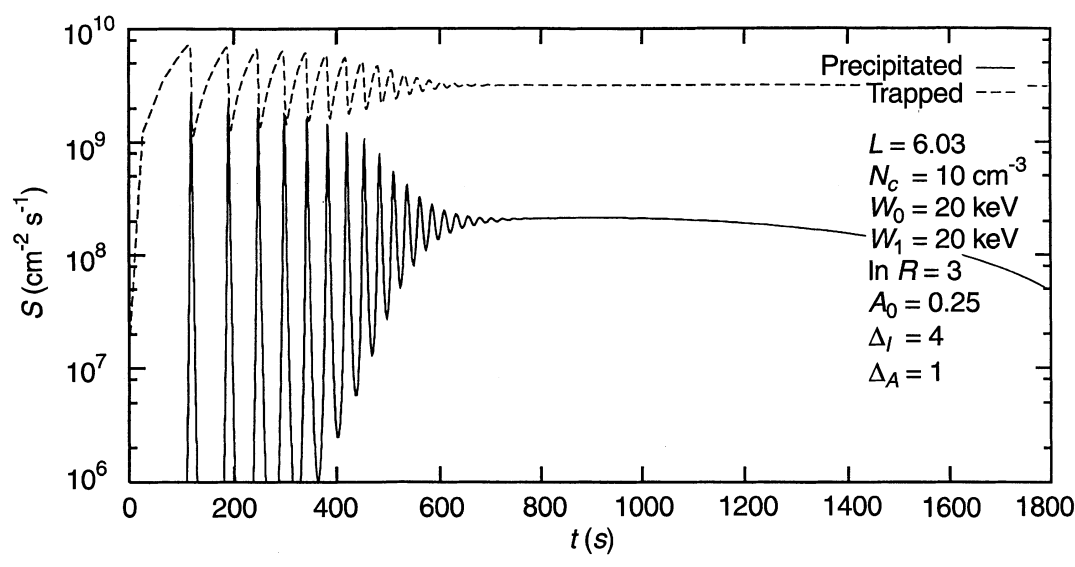

Fig. 5. Same as in Fig. 4 but for stronger and shorter injection with smaller variation of the pitchangle distribution in the source; $T_{I}=1800 \mathrm{~s}$ 
Table 1. Parameters of pulsating electron precipitation events of 17 December 1990, analyzed by Manninen et al. (1996); Lyubchich et al. (1996). Properties of auroral pulsations have been obtained from EISCAT data, and characteristics of energetic electron injection, from LANL satellites and CRRES

\begin{tabular}{lll}
\hline Duration of injection event & $\begin{array}{l}\text { Event } 1 \\
\approx 50 \mathrm{~min}\end{array}$ & $\begin{array}{l}\text { Event } 2 \\
\text { min }\end{array}$ \\
\hline $\begin{array}{l}\text { Energy dispersion of energetic particles } \\
\text { Pitch angle distribution }\end{array}$ & $\begin{array}{l}\text { Significant } \\
\text { Evolved from the pancake shape } \\
\left.\text { (peaked at } 90^{\circ}\right) \text { to butterfly and back } \\
\text { during the event }\end{array}$ & $\begin{array}{c}\text { Almost absent } \\
\text { Pancake shape all the time }\end{array}$ \\
$\begin{array}{l}40 \text { min }(02: 30-03: 10 \text { UT): All the duration } \\
\text { of the event; period increased in the middle }\end{array}$ & $\begin{array}{c}7 \text { min (03:19-03:26 UT): Only during } \\
\text { the 1st half of the event }\end{array}$ \\
\hline
\end{tabular}

in case of event 1, exhibiting significant dispersion, injection region is located at some distance from the region of pulsating precipitation.

Figure 4 represents a localized injection which occurred at some distance from the observation point; substantial variation of the pitch-angle distribution due to pitch-angle dependence of the drift velocity is expected in this case (we have chosen $\Delta_{A}=4$ ), and such a variation has been indeed recorded (Lyubchich et al., 1996). According to the data, the maximum of the "sloshing" component of the pitch-angle distribution occurred near the maximum density of the cloud. The same behaviour is chosen for the calculations. During that injection, one could observe pulsations during the whole event, and their period increased near the maximum density of the energetic electron cloud. Such an evolution is in agreement with the numerical results shown in Fig. 4.

Parameters chosen for the calculations represented in Fig. 5 correspond to the case of the shorter and sharper injection (event 2 in Table 1) which probably occurred close to the instability region (this can be concluded from absence of energy and pitch-angle dispersion). For that case, pulsations were seen only at the beginning of the event (Manninen et al., 1996). Similar behaviour has been obtained in numerical calculations shown in Fig. 5. The related features of the chosen parameters are (a) shorter time scale; (b) larger relative variation of the energetic particle flux $\left(\Delta_{I}=4\right)$; and (c) smaller variation of the anisotropy factor $A\left(\Delta_{A}=1\right)$.

\section{Conclusion}

We can conclude that the simple self-consistent model of a magnetosphere cyclotron maser used in this work permitted us to explain qualitatively different temporal patterns of auroral pulsations observed in experiment; that explanation is consistent with the parameters of cold and energetic plasma that follow from the data.

The simplified Eq. (1) is known to be violated by the moderate pitch-angle diffusion regime. An important task for future work is to compare the results reported here with those from a more sophisticated model. In particular, calculation of full distribution over pitch angle and energy would make possible a more detailed comparison with observations. However, such calcula- tions will be rather complicated, so using the simplified equations like those studied in this work seems to be important for initial choice of parameters as well as for investigating some common features of energetic particle precipitation.

Acknowledgements. The authors are grateful to Lev Zeleny for valuable comments. This work was supported in part by the INTAS grant 94-2753 and by the Russian Foundation for Basic Research, grant 96-02-16473a.

Topical Editor K.-H. Glassmeier ank, L.M. Zelenyi for his help in evaluating this paper.

\section{References}

Bespalov, P. A., Self-modulation of the plasma cyclotron maser emission, Pis'ma Zh. Eksp. Teozet. Fiz., 33, 192-195, 1981.

Bespalov, P. A., and V. Y. Trakhtengerts, On nonlinear oscillatory processes in the Earth magnetosphere, Izv. Vuzov - Radiofiz., 19, 801-811, 1976.

Bespalov, P. A., and V. Y. Trakhtengerts, The cyclotron instability in the Earth radiation belts, in Reviews of Plasma Physics, Ed. M. A. Leontovich, volume 10, 155-192, Plenum, New York, 1986.

Davidson, G. T., Self-modulated VLF wave-electron interactions in the magnetosphere: a cause of auroral pulsations, J. Geophys. Res., 84, 6517-6523, 1979.

Davidson, G. T., and Y. T. Chiu, A closed nonlinear model of wave-particle interactions in the outer trapping and morningside auroral regions, J. Geophys. Res., 91, 13705-13710, 1986.

Demekhov, A. G., On the role of the loss cone in the formation of the spike-like regime of the whistler cyclotron instability, Geomagn. Aeron., 31, 1099-1101, 1991.

Demekhov, A. G., and V. Y. Trakhtengerts, A mechanism of formation of pulsating aurorae, J. Geophys. Res., 99, 58315841, 1994.

Lyubchich, A. A., A. G. Yahnin, E. E. Titova, V. Y. Trakhtengerts, A. G. Demekhov, R. D. Belian, J. Manninen, T. Turunen, and R. Rasinkangas, Longitudinal drift and precipitation of the energetic electrons during the substorm, in Proc. XIX Seminar on Auroral Phenomena, 63-66, Apatity, Russia, PGI, 1996.

Manninen, J., T. Turunen, A. A. Lubchich, E. E. Titova, and T. A. Yahnina, Relations of VLF emissions to impulsive electron presipitation measured by EISCAT radar in the morning sector of auroral oval, J. Atmos. Terr. Phys., 58, 97-106, 1996.

Sandahl, I., Pitch angle scattering and pitch angle precipitation in a pulsating aurora - an experimental study, Techni. Rep. 185, Kiruna Geophys. Inst., Kiruna, Sweden, 1984.

Schulz, M., Particle lifetimes in strong diffusion, Astrophys. Space Sci., 31, 37-41, 1974.

Trakhtengerts, V. Y., V. R. Tagirov, and S. A. Chernous, Flow cyclotron maser and impulsive VLF emissions, Geomagn. Aeron., 26, 99-106, 1986. 